\title{
ALMOST EVERYWHERE CONVERGENCE OF POISSON INTEGRALS ON TUBE DOMAINS OVER CONES
}

\author{
BY \\ NORMAN J. WEISS $\left({ }^{1}\right)$
}

1. Introduction. An important class of domains of several complex variables consists of tube domains $T_{D}$ over open convex cones $D$. These domains are analogous to the upper half plane in classical complex analysis, and there is a natural analog to the Poisson kernel which reproduces the real parts of holomorphic functions on $T_{D}$ from their boundary values. The purpose of this paper is to generalize the theorem of Fatou which states that the Poisson integral of an integrable function $f$ on the line converges to $f$ almost everywhere. A partial generalization is found in the case of tube domains over cones which are domains of positivity (to be defined in $\$ 2$ ); these tube domains are holomorphically equivalent to certain of the bounded symmetric domains classified by E. Cartan.

By an open convex cone, we mean an open, convex subset $D$ of Euclidean $n$-space $E_{n}$ such that if $x \in D$ and $\lambda>0$, then $\lambda x \in D,-\lambda x \notin D$. The tube domain $T_{D}$ over $D$ is the subset of complex $n$-dimensional space consisting of vectors of the form $z=x+i y, x \in E_{n}, y \in D$. We say that a holomorphic function $F$ on $T_{D}$ is of the class $H^{p}, 0<p<\infty$, if $\sup _{y \in D} \int_{E_{n}}|F(x+i y)|^{p} d x<\infty$.

There is a natural generalization of the Poisson kernel for the upper half plane (the tube domain over the positive half line) to a function $P_{y}(x)$ on $E_{n}$, indexed by $y \in D$. (The formula for $P_{y}(x)$ is given in $\S 2$.) It is shown in the paper of E. Stein, G. Weiss and M. Weiss [14] that $P_{y}(x)$ has these important properties:

(1) If $F \in H^{2}\left(T_{D}\right)$ and $F(x)=\lim _{y \rightarrow 0} F(x+i y)$, where the limit is in the $L^{2}\left(E_{n}\right)$ norm (and can easily be shown to exist), then $\operatorname{Re}[F(x+i y)]=\int_{E_{n}} P_{y}(x-t) \operatorname{Re}[F(t)]$.

(2) $P_{y}(x)$ is an approximation to the identity, i.e., for all $y \in D, \int_{E_{n}} P_{y}(x) d x=1$, and for all $\delta>0, \lim _{y \rightarrow 0} \int_{|x|>\delta} P_{y}(x) d x=0$.

Our interest in this paper centers about the boundary behavior of functions $u(x+i y)$ on $T_{D}$ as $y \rightarrow 0$. (Points of the form $x+i \cdot 0, x \in E_{n}$, make up the distinguished boundary of $T_{D}$ in the sense of Bergman.) It will be useful to define the notion of restricted convergence of $y \in D$ to the origin; we say that $y \rightarrow 0$ restrictedly if there is a constant $a$ such that

$$
|y| / d(y, \partial D)<a
$$

Received by the editors November 16, 1966.

( $\left.{ }^{1}\right)$ This paper includes part of the author's Doctoral disseriation at Princeton University, prepared under the supervision of Professor E. M. Stein while the author was supported by a National Science Foundation Graduate Fellowship. This research was partially supported by Air Force Contract AF 49638-1529. 
that is, if $y$ does not come "too close" to the boundary $\partial D$. Convergence of $y$ to the origin without this condition is referred to as unrestricted convergence.

It is shown in [14] that if $F \in H^{p}\left(T_{D}\right), 0<p<\infty$, then $F(x+i y)$ converges a.e. on $E_{n}$ to its $L^{p}$ boundary value $F(x)$ as $y \rightarrow 0$ restrictedly; the same, of course, holds for $f=\operatorname{Re} F$. Suppose now that $f$ is an arbitrary real-valued function in $L^{p}\left(E_{n}\right)$, $1 \leqq p \leqq \infty$, and set

$$
u(x+i y)=\int_{E_{n}} P_{y}(x-t) f(t) d t .
$$

It is natural to ask whether $u(x+i y)$ converges to $f(x)$ a.e. on $E_{n}$ as $y \rightarrow 0$. (It is not necessarily true that $f$ is the boundary value of the real part of a function holomorphic on $T_{D}$. See, for instance, [11].)

In the case when $D$ is an octant, i.e.,

$$
D=\left\{\left(y_{1}, \ldots, y_{n}\right): y_{1}>0, \ldots, y_{n}>0\right\},
$$

in which case $T_{D}$ is the Cartesian product of $n$ half planes, the following is known:

(a) If $f \in L^{p}\left(E_{n}\right), p>1$, or even if $f \in L\left(\log ^{+} L\right)^{n-1}$ locally, then $u(x+i y) \rightarrow f(x)$ a.e. as $y \rightarrow 0$ unrestrictedly. (This is a corollary of the argument used by Jessen, Marcinkiewicz and Zygmund [5] to prove strong differentiability of multiple integrals.)

(b) If $f \in L^{1}\left(E_{n}\right)$, then $u(x+i y) \rightarrow f(x)$ a.e. on $E_{n}$ as $y \rightarrow 0$ restrictedly. (This is a result of Marcinkiewicz and Zygmund [12].)

The situation is not so nice when $D$ is a more general cone, and, in particular, a "smoother" cone. In fact, it is shown in [14] that if $D$ is the forward light cone $C_{n} \subset E_{n}$, (to be defined in $\$ 4$ ) then for every $n \geqq 3$ and $p<2 n / 3$, there exists $f \in L^{p}\left(E_{n}\right)$ such that $\lim \sup _{y \rightarrow 0} u(x+i y)=\infty$ a.e. on $E_{n}$, where $y$ is allowed to converge unrestrictedly. In this paper, we show that a result like (b) holds when $D$ is a domain of positivity.

In $\$ 2$, we give the definition of a domain of positivity and discuss some of its properties. In $\$ 4$ we show that if $D=C_{n}$, the forward light cone in $E_{n}$, then $u(x+i y) \rightarrow f(x)$ a.e. as $y \rightarrow 0$ restrictedly, if $f \in L^{p}$ for any $p>1$, or even if $f$ is locally in $L\left(\log ^{+} L\right)$. As will be remarked, this is a strong indication that the condition $f \in L^{1}$ is sufficient to guarantee restricted a.e. convergence of $u(x+i y)$ to $f(x)$. We also show that the condition $f \in L^{1}$ does suffice in the nontrivial case when $n=3$. In $\$ \S 5-7$, we use the theory of Jordan algebras to deduce the former result when $D$ is an arbitrary domain of positivity.

The method of proof in each case is to estimate $u(x+i y)$ in terms of certain maximal averages of $f$, to use the so-called method of rotations to relate these maximal functions to maximal functions over rectangular sets in $E_{n}$, and then to use the properties of the latter set down in $\S 3$.

To conclude this section, I would like to express my deepest gratitude to Professor E. M. Stein, who suggested the topic of this paper to me and gave his time freely for many invaluable conversations. 
2. The Poisson kernel; domains of positivity. In this section, we discuss the Poisson kernel for tube domains over cones, define domains of positivity, and give some of their properties.

In his paper [1], Bochner defined the analog of the Cauchy kernel for the tube domain over an open convex cone, $D$. Defining the dual cone,

$$
D^{*}=\left\{t \in E_{n}: t \cdot y \geqq 0, \text { all } y \in D\right\}
$$

(and assuming that $D^{*}$ has nonempty interior), the Cauchy kernel is given by

$$
K(x+i y)=K(z)=\int_{D^{*}} e^{2 \pi i z \cdot t} d t .
$$

The function $K(z)$ is easily seen to be holomorphic on $T_{D}$. Moreover, if $F(z) \in H^{2}\left(T_{D}\right)$ and $F(x)$ is its $L^{2}$ boundary value, then

$$
F(x+i y)=\int_{E_{n}} K(x-t+i y) F(t) d t .
$$

The Poisson kernel is given by

$$
P_{y}(x)=|K(x+i y)|^{2} / K(2 i y) .
$$

The properties (1) and (2) of the Poisson kernel which were given in $\S 1$ hold when $D$ is an arbitrary open convex cone. (The property (1) is a consequence of formulas (2) and (3) above.) More information about the Poisson kernel is available if $D$ is taken to have additional properties.

A cone $D$ is said to be a domain of positivity if

(1) $D$ is self-dual, i.e., $D^{*}=\bar{D}$.

(2) $D$ is homogeneous, i.e., there is a group $G$ of linear automorphisms of $E_{n}$ which takes $D$ into itself and acts transitively on $D$.

Domains of positivity were studied initially by Koecher [7]. Many of the major properties of tube domains over domains of positivity are given in the paper of Rothaus [13]. Function analysis on tubes over domains of positivity is developed in the paper of Koranyi [9].

It is shown in [10] that tube domains over domains of positivity are holomorphically equivalent to bounded symmetric homogeneous domains. As is mentioned in [10], it can be shown further that the class of bounded domains equivalent to these tube domains is exactly the class of so-called tube type, consisting of domains having a distinguished boundary whose dimension is half that of the domain.

It is of interest that Koranyi [9] found Poisson integrals, i.e., functions of the form $u(x+i y)=P_{y} * f(x)$ on tube domains over domains of positivity to satisfy the equation $\Delta u=0$ with respect to the Laplace-Beltrami operator arising from the riemannian structure on the tube domain.

We conclude this section by stating two conventions that will prevail for the remainder of the paper.

(1) $|E|$ denotes the Lebesgue measure of a (measurable) subset of $E_{n}$. 
(2) Functions on $E_{n}$ are assumed to be nonnegative. It will be clear that this involves no loss of generality.

3. Rectangular maximal functions. In this section are collected several results about maximal functions over rectangular sets in $E_{n}$. The proof of part (a) of Lemma 3.2 reveals the obstacle standing in the way of a proof of a.e. convergence for Poisson integrals of $L^{1}$ functions on tube domains over arbitrary domains of positivity.

The results we need are $n$-dimensional variants of the following well-known theorem of Hardy and Littlewood [4]. Recall that all functions are to be taken as nonnegative.

THEOREM 3.1. Let $f$ be a function on $(-\infty, \infty)$, and set

$$
\bar{f}(x)=\sup _{\varepsilon>0}(2 \varepsilon)^{-1} \int_{x-\varepsilon}^{x+\varepsilon} f(x-t) d t .
$$

Then there exist constants $A^{\prime}, A_{p}, 1 \leqq p \leqq \infty$, independent of $f$, such that

(a) For all $s>0,|\{x: \bar{f}(x)>s\}|<A^{\prime} s^{-1}\|f\|_{1}$.

(b) $\|\bar{f}\|_{p} \leqq A_{p}\|f\|_{p}, 1<p \leqq \infty$.

(c) If $f$ is supported on $[-1,1]$, then

$$
\int_{-1}^{1} \bar{f}(x) d x \leqq A_{1} \int_{-1}^{1} f(x) \log ^{+} f(x) d x+A_{1},
$$

where $\log ^{+} u=0, u<1, \log ^{+} u=\log u, u \geqq 1$.

In what follows, $x \in E_{n}$ will have components $\left(x_{1}, \ldots, x_{n}\right)$ and $(j)$ will stand for an $n$-tuple $\left(j_{1}, \ldots, j_{n}\right)$ of nonnegative integers.

Definition. For $\varepsilon>0, R_{(j)}^{\varepsilon}=\left\{x \in E_{n}:\left|x_{i}\right| \leqq 2^{j_{i}} \varepsilon, i=1, \ldots, n\right\}$.

For a function $f$ on $E_{n}$,

$$
\begin{aligned}
& f_{(j)}^{\varepsilon}(x)=\left|R_{(j)}^{\varepsilon}\right|^{-1} \int_{R_{(j)}^{\varepsilon}} f(x-t) d t . \\
& \bar{f}_{(j)}(x)=\sup _{\varepsilon>0} f_{(j)}^{\varepsilon}(x) .
\end{aligned}
$$

LEMMA 3.2. (a) $\left|\left\{x \in E_{n}: \bar{f}_{(j)}(x)>s\right\}\right|<B^{\prime} s^{-1}\|f\|_{1}$.

(b) $\left\|\bar{f}_{(j)}(x)\right\|_{p} \leqq B_{p}\|f(x)\|_{p}, 1<p \leqq \infty$.

In each case, the constant is independent of $f$ and of $(j)$.

The proof of part (b) follows from repeated application of Theorem 3.1(b).

The proof of (a) is in the paper of Marcinkiewicz and Zygmund [12], and is the crux of their proof of restricted a.e. convergence for the Poisson integral of an $L^{1}$ function on $T_{\text {octant }}$. It is based on a Vitali-type covering argument, which in turn rests upon a crucial fact about rectangular sets, which we call

Property A. Let $R$ be a rectangular set in $E_{n}$, and let $\bar{R}$ be the union of all sets congruent to $R$ and oriented like $R$ which meet $R$. 
Then $|\bar{R}|<c|R|$, where $c$ is a constant depending only on $n$. (In fact, $c$ can be any number larger than $3^{n}$.)

As will be seen in $\$ \$ 4$ and 6, estimates for the Poisson integrals of functions on the tube domains we consider involve maximal functions over sets which do not satisfy Property A. These maximal functions may not satisfy the conclusion of 3.2(a), which is needed in the proof of restricted a.e. convergence for Poisson integrals of $L^{1}$ functions.

The result we substitute for 3.2(a) is the $n$-dimensional variant of 3.1(c). It is proved here as a special case of the interpolation theorem of Marcinkiewicz. (See [15, II, p. 118].)

LEMMA 3.3. Suppose that $f$ is supported on the unit cube $I_{n}$ in $E_{n}$.

Then $\int_{I_{n}} \bar{f}_{(j)}(x) d x \leqq B_{1} \int_{I_{n}} f(x) \log ^{+} f(x) d x+B_{1}$, where $B_{1}$ depends only on $n$.

Proof. We show that $B_{1}$ depends only on the bounds $B^{\prime}$ and $B_{2}$ in 3.2. (The choice of $p=2$ is inconsequential.)

Writing $\bar{f}_{(j)}=F$, it follows in particular from 3.2(b) that

$$
m(F)(s)=|\{x: F(x)>s\}|<\left(B_{2} / s\right)^{2} \int_{I_{n}} f(x)^{2} d x .
$$

For each value of $s>0$, let $f=f_{1}+f_{2}$, where

$$
\begin{aligned}
f_{1}(x) & =f(x), & & f(x)>s / 2, \\
& =0, & & f(x) \leqq s / 2 .
\end{aligned}
$$

Set $\quad F_{1}=\bar{f}_{1_{(\jmath)},}, \quad F_{2}=\bar{f}_{2_{(\jmath)}} . \quad$ Clearly, $\quad F \leqq F_{1}+F_{2}, \quad$ and so $m(F)(r) \leqq m\left(F_{1}\right)(r / 2)$ $+m\left(F_{2}\right)(r / 2)$ for all $r>0$ and, in particular, for $r=s$.

Now $\int_{I_{n}} F \leqq\left(\int_{F>1} F\right)+2^{n}$, and the first term is equal to

$$
\int_{1}^{\infty} m(F)(s) d s \leqq J_{1}+J_{2}=\int_{1}^{\infty} m\left(F_{1}\right)(s / 2) d s+\int_{1}^{\infty} m\left(F_{2}\right)(s / 2) d s,
$$

where the decomposition $f=f_{1}+f_{2}$ depends in each case upon $s$.

From 3.2(a), it follows that

$$
\begin{aligned}
J_{1} \leqq B^{\prime} \int_{1}^{\infty} 2 s^{-1} \int_{I_{n}} f_{1}(x) d x d s & =2 B^{\prime} \int_{1}^{\infty} s^{-1} \int_{f>s / 2} f(x) d x d s \\
& =2 B^{\prime} \int_{f>1 / 2} f(x) \int_{1}^{2 f(x)} s^{-1} d s d x \\
& =2 B^{\prime} \int_{f>1 / 2} f(x) \log [2 f(x)] d x \\
& \leqq c \int_{I_{n}} f(x) \log ^{+} f(x) d x+c^{\prime}
\end{aligned}
$$

where we have used the inequality: $u \leqq u \log ^{+} u+1, u \geqq 0$. 
On the other hand, it follows from (1) that

$$
\begin{aligned}
J_{2} & \leqq\left(2 B_{2}\right)^{2} \int_{1}^{\infty} s^{-2} \int_{f<s / 2} f(x)^{2} d x d s \\
& =\left(2 B_{2}\right)^{2}\left[\int_{f<1 / 2} f(x)^{2} \int_{1}^{\infty} s^{-2} d s d x+\int_{f>1 / 2} f(x)^{2} \int_{2 f(x)}^{\infty} s^{-2} d s d x\right] \\
& =\left(2 B_{2}\right)^{2}\left[\int_{f<1 / 2} f(x)^{2} d x+(1 / 2) \int_{f>1 / 2} f(x) d x\right] \\
& \leqq C \int_{I_{n}} f(x) \log ^{+} f(x) d x+C^{\prime} .
\end{aligned}
$$

This completes the proof of Lemma 3.3.

Remarks. Part (b) of Lemma 3.2 can be proved by the same method as Lemma 3.3, using the obvious fact that $\left\|\bar{f}_{(j)}\right\|_{\infty} \leqq\|f\|_{\infty}$, together with 3.2(a). Also, 3.3 follows from a well-known result of Wiener [16, Theorem $\left.V^{\prime}\right]$ once one makes the observation that rectangular sets satisfy Property A and that one can thus replace the spheres in Lemma $C^{\prime}$ of [16] with rectangular sets whose sides are in fixed ratio to one another.

4. Almost everywhere convergence on $T_{C_{n}}$. In this section we will always write

and

$$
x=\left(x_{1}, \ldots, x_{n}\right)=\left(x_{1}, r\right), \quad r \in E_{n-1},
$$

$$
(x, y)=x_{1} y_{1}-x_{2} y_{2}-\cdots-x_{n} y_{n} .
$$

With this notation, we can define the forward light cone in $E_{n}$ as

$$
C_{n}=\left\{x=\left(x_{1}, \ldots, x_{n}\right): x_{1}>0,(x, x)>0\right\}=\left\{x=\left(x_{1}, r\right): x_{1}>|r|\right\} .
$$

$C_{n}$ is clearly a domain of positivity with the transformation group

$$
G=\left\{T \in G L(n):(T x, T y)=C_{T}(x, y)\right\} .
$$

We begin by stating the main results of this section.

THEOREM 4.1. Let $f$ be a function on $E_{n}$ and let

$$
u(x+i y)=P_{y} * f(x)=\int_{E_{n}} P_{y}(x-t) f(t) d t, \quad y \in C_{n}
$$

be the Poisson integral of $f$ on $T_{C_{n}}$.

Suppose further that one of the following holds:

(a) $f \in L^{1}\left(E_{n}\right)$ and $\int_{B} f(x) \log ^{+} f(x) d x<\infty$ whenever $B \subset E_{n}$ is bounded.

(b) $f \in L^{p}\left(E_{n}\right)$ for some $p, 1<p \leqq \infty$.

Then $u(x+i y) \rightarrow f(x)$ a.e. on $E_{n}$ as $y \rightarrow 0$ restrictedly.

THEOREM 4.2. When $n=3$, the condition $f \in L^{1}\left(E_{n}\right)$ is also sufficient for the restricted a.e. convergence of $u(x+i y)$ to $f(x)$. 
Theorem 4.1 is a special case of the more general Theorem 6.1, which gives the same result for tube domains over arbitrary irreducible domains of positivity. A separate proof of the former is included to make the general result more easily understandable to the nonspecialist in Jordan algebras, as well as to provide a basis for the proof of Theorem 4.2.

To establish Theorem 4.1, we estimate $P_{y} * f$ in terms of certain maximal functions, use the method of rotations to relate these maximal functions to the rectangular maximal functions of $\S 3$, and then apply the results of $\S 3$.

The proof of Theorem 4.2 uses the same estimates, but substitutes a "discrete" version of the method of rotations, which seems useful only in the case $n=3$.

Now let

$$
y=\left(y_{1}, R\right) \in C_{n}, \quad \text { i.e., } \quad y_{1}>|R| \text {. }
$$

We notice that the condition that $y \rightarrow 0$ restrictedly is equivalent to:

$$
|R|<a y_{1}
$$

where $a$ is a constant and $a<1$. To see this, it is enough to take $y=\left(y_{1}, y_{2}, 0, \ldots, 0\right)$. The condition of restricted convergence is then

$$
d\left(y, \partial C_{n}\right) /|y|=\left(\frac{1}{2}\right)(2)^{1 / 2}\left(y_{1}-\left|y_{2}\right|\right) /|y|>\delta>0,
$$

which is clearly equivalent to (1).

In his paper [1], Bochner showed that the Cauchy kernel for $C_{n}$ is given by analytic continuation of the formula

$$
K(i y)=c_{n}^{\prime}(y, y)^{-n / 2} .
$$

It is immediate that the Poisson kernel for $C_{n}$ is given by:

$$
P_{y}(x)=c_{n}(y, y)^{n / 2} /\left[((x, x)-(y, y))^{2}+4(x, y)^{2}\right]^{n / 2} .
$$

Lemma 4.3. Suppose that $y=\left(y_{1}, R\right)$ satisfies (1) and that $x=\left(x_{1}, r\right) \in E_{n}$.

Then $P_{y}(x) \leqq A P_{y_{1}}(x)$, where $y_{1}$ refers to the vector $\left(y_{1}, 0, \ldots, 0\right)$, and $A$ depends only on $a$ and $n$.

This is a special case of Lemma 6.3; the proof in the specific case at hand is tedious, but straightforward. It consists of applying the formulae (1) and (2) separately to the three cases: (a) $|x|$ is small compared to $|y|$; (b) $|x|$ is large and $(x, x)^{1 / 2}$ is small compared to $|y|$; (c) $|x|$ and $(x, x)^{1 / 2}$ are large compared to $|y|$.

On the basis of the lemma, and with a slight abuse of notation, we will from now on write $y=(y, 0, \ldots, 0)$ for $y \in C_{n}$.

Definition. For $\varepsilon>0, i \leqq j, i=0,1,2, \ldots$,

$$
B_{i, j}^{\varepsilon}=\left\{x=\left(x_{1}, r\right) \in E_{n}:\left|x_{1}\right|+|r| \leqq 2^{j} \varepsilon,|| x_{1}|-| r|| \leqq 2^{i} \varepsilon\right\}
$$


For a function $f$ on $E_{n}$,

$$
\begin{aligned}
& f_{i, j}^{\varepsilon}(x)=\left|B_{i, j}^{\varepsilon}\right|^{-1} \int_{B_{i, j}^{\varepsilon}} f(x-t) d t . \\
& f_{i, j}^{*}(x)=\sup _{\varepsilon>0} f_{i, j}^{\varepsilon}(x) .
\end{aligned}
$$

We notice, and this will be important, that $B_{i, j}^{\varepsilon}$ is obtained by rotating about the $x_{1}$ axis (over $S^{n-2}$ ) the two-dimensional rectangle

$$
\tilde{R}_{i, j}^{\varepsilon}=\left\{\left(t_{1}, t_{2}\right) \in E_{2}:\left|t_{1}+t_{2}\right| \leqq 2^{j} \varepsilon,\left|t_{1}-t_{2}\right| \leqq 2^{i} \varepsilon\right\} .
$$

We see also that $\widetilde{R}_{i, j}^{\varepsilon}$ is (to within a constant factor) the rectangle $R_{i, j}^{\varepsilon}$ of $\$ 3$, rotated about the origin in $E_{2}$ by $45^{\circ}$.

Lemma 4.4. $P_{y} * f(x) \leqq A \sum_{i \leqq j} 2^{-[j+(n-1) i]} f_{i, j}^{*}(x)$, where $A$ is a constant independent of $i, j, x, y$, and $f$.

Proof. Notice first that $\left|B_{i, j}^{\varepsilon}\right| \sim \varepsilon^{n} 2^{i+(n-1) j}$, as is clear from the immediately preceding remarks. (By $F(i, j) \sim G(i, j)$, we mean that there exist constants $a_{1}$ and $a_{2}$ independent of $i$ and $j$ such that $a_{1} F(i, j) \leqq G(i, j) \leqq a_{2} F(i, j)$.)

Recall that we assume $y=(y, 0, \ldots, 0)$. For $y$ in this form, and $t=\left(t_{1}, \rho\right)$,

$$
P_{y}(t)=c_{n} y^{n} /\left[y^{4}+2 y^{2}\left(t_{1}^{2}+|\rho|^{2}\right)+(t, t)^{2}\right]^{n / 2} .
$$

Now

where

$$
P_{y} * f(x)=\int_{E_{n}} P_{y}(t) f(x-t) d t=\int_{A_{1}}+\int_{A_{2}}+\int_{A_{3}},
$$

$$
\begin{aligned}
& A_{1}=\left\{t:\left|t_{1}\right|+|\rho| \leqq y\right\}, \\
& A_{2}=\left\{t:\left|t_{1}\right|+|\rho| \geqq y,|| t_{1}|-| \rho|| \leqq y\right\}, \\
& A_{3}=\left\{t:\left|t_{1}\right|+|\rho| \geqq y,|| t_{1}|-| \rho|| \geqq y\right\} .
\end{aligned}
$$

The following estimates of $P_{y}(t)$ are now trivial consequences of (3):

$$
\begin{aligned}
P_{y}(t) & \leqq c_{n} y^{-n}, & & t \in A_{1}, \\
& \leqq c_{n} /\left[2^{n / 2}\left(t_{1}^{2}+|\rho|^{2}\right)^{n / 2}\right], & & t \in A_{2}, \\
& \leqq c_{n} y^{n} /(t, t)^{n}, & & t \in A_{3} .
\end{aligned}
$$

The situation on $A_{1}$ is easiest, since

$$
\int_{A_{1}} P_{y}(t) f(x-t) d t \leqq c_{n} y^{-n} \int_{A_{1}} f(x-t) d t \leqq A f_{0}^{*}, 0(x) .
$$

Now for $i, j=1,2, \ldots, i \leqq j$, define

$$
F_{i, j}^{y}=\left\{t: 2^{j-1} y \leqq\left|t_{1}\right|+|\rho| \leqq 2^{j} y, 2^{i-1} y \leqq \| t_{1}|-| \rho|| \leqq 2^{i} y\right\},
$$

and notice that $F_{i, j}^{y} \subset B_{i, j}^{y}$. We have $A_{3}=\bigcup_{i \leqq j} F_{i, j}^{y}$, and if $t \in F_{i, j}^{y}$, then

$$
P_{y}(t) \leqq c_{n} y^{n} /(t, t)^{n} \leqq c\left(2^{i+j} y\right)^{-n} .
$$


Therefore, $t \in F_{i, j}^{y}$ implies that $P_{y}(t)\left|B_{i, j}^{y}\right| \leqq 2^{-[j+(n-1) i]} A$, and so

$$
\begin{aligned}
\int_{A_{3}} P_{y}(t) f(x-t) d t & \leqq c \sum_{i \leqq j}\left(2^{i+j} y\right)^{-n} \int_{F_{i, j}^{v}} f(x-t) d t \\
& \leqq A \sum_{i \leqq j} 2^{-[j+(n-1) i]}\left|B_{i, j}^{y}\right|^{-1} \int_{B_{i, j}^{y}} f(x-t) d t \\
& \leqq A \sum_{i \leqq j} 2^{-[j+(n-1) i]} f_{i, j}^{*}(x) .
\end{aligned}
$$

Finally, for $j=1,2, \ldots$, define

$$
F_{0, j}^{y}=F_{j}^{y}=\left\{t: 2^{j-1} y \leqq\left|t_{1}\right|+|\rho| \leqq 2^{j} y,|| t_{1}|-| \rho|| \leqq y\right\} .
$$

Now $A_{2}=\bigcup_{j} F_{j}^{y}$, and if $t \in F_{j}^{y}$, then

$$
P_{y}(t) \leqq c /\left(t_{1}^{2}+|\rho|^{2}\right)^{n / 2} \leqq c^{\prime} 2^{-n j} y^{-n},
$$

and so it follows as above that

$$
\int_{A_{2}} P_{y}(t) f(x-t) d t \leqq A \sum_{j} 2^{-j} f_{0, j}^{*}(x)
$$

The proof of Lemma 4.4 is thus complete.

The properties of the maximal functions $f_{i, j}^{*}$ will be deduced from the properties of the rectangular maximal functions $\bar{f}_{i, j}$ of $\S 3$ by means of the method of rotations used by Calderón and Zygmund in singular integral theory [3].

Lemma 4.5. Suppose that $f$ is supported on the unit cube $I_{n}$ in $E_{n}$, and that $f_{i, j}^{*}$ is the maximal function of Lemma 4.4. Then

$$
\int_{I_{n}} f_{i, j}^{*}(x) d x \leqq A \int_{I_{n}} f(x) \log ^{+} f(x) d x+A,
$$

where $A$ is independent of $i, j$, and $f$.

Proof. Recalling the definitions of $B_{i, j}^{\varepsilon}$ and $\tilde{R}_{i, j}^{\varepsilon}$ preceding 4.4 , and that $\left|B_{i, j}^{\varepsilon}\right|$ $\sim \varepsilon^{n} 2^{[(n-1) j+i]}$, we have

$$
f_{i, j}^{*}(x)=f_{i, j}^{*}\left(x_{1}, r\right)=\sup _{\varepsilon>0}\left|B_{i, j}^{\varepsilon}\right|^{-1} \int_{B_{i, j}^{\varepsilon}} f(x-t) d t
$$

$$
\leqq \sup _{\varepsilon<0}\left(\omega_{n-2}\right)^{-1} \int_{S^{n-2}} d y^{\prime}\left[c \varepsilon^{-n} 2^{-[(n-1) j+i]} J_{i, j} f\left(x_{1}, r ; y^{\prime}\right)\right]
$$

where $J_{i, j} f\left(x_{1}, r ; y^{\prime}\right)=J_{i, j} f\left(x ; y^{\prime}\right)=\int_{\tilde{R}_{i, j}^{\varepsilon}} f\left(x_{1}-t_{1}, r-|\rho| y^{\prime}\right)|\rho|^{n-2} d|\rho| d t_{1}$. (We again write $t=\left(t_{1}, \rho\right)$.)

For $\left(t_{1},|\rho|\right) \in \tilde{R}_{i, j}^{\varepsilon},|\rho|^{n-2} \leqq 2^{(n-2) j} \varepsilon^{n-2}$, so the inner term in (4) is dominated by

$$
c^{\prime} 2^{-(j+i)} \varepsilon^{-2} \int_{\tilde{R}_{i, j}^{\varepsilon}} f\left(x_{1}-t_{1}, r-\rho y^{\prime}\right) d \rho d t_{1} .
$$


Denote by $\bar{f}_{i, j}\left(x ; y^{\prime}\right)=\bar{f}_{i, j}\left(x_{1}, r ; y^{\prime}\right)$ the supremum over $\varepsilon$ of $(5)$, and notice that $\left|\tilde{R}_{i, j}^{\varepsilon}\right| \sim \varepsilon^{2} 2^{j+i}$. Thus $\bar{f}_{i, j}\left(x_{1}, r ; y^{\prime}\right)$ is a rectangular maximal function in $E_{2}$ for each fixed $y^{\prime} \in S^{n-2}$. (It is the maximal average of the function $f$ taken over 2-dimensional rectangles "pointed" in the direction of $y^{\prime}$.)

Applying Lemma 3.3, it follows that

$$
\begin{aligned}
& \int_{\left(x_{1}-t_{1}, r-|\rho| y^{\prime}\right) \in I_{n}} \bar{f}_{i, j}\left(x_{1}-t_{1}, r-|\rho| y^{\prime} ; y^{\prime}\right) d t_{1} d|\rho| \\
& \leqq A^{\prime} \int_{\cdots \in I_{n}} f\left(x_{1}-t_{1}, r-|\rho| y^{\prime}\right) \log ^{+} f\left(x_{1}-t_{1}, r-|\rho| y^{\prime}\right) d|\rho| d t_{1}+A^{\prime}
\end{aligned}
$$

Integrating now over all planes parallel to the one determined by $x_{1}, r$ and $y^{\prime}$, i.e., along the orthogonal complement, we have

$$
\int_{I_{n}} \bar{f}_{i, j}\left(x ; y^{\prime}\right) d x \leqq A \int_{I_{n}} f(x) \log ^{+} f(x) d x+A .
$$

Now integrating in (6) over all $y^{\prime} \in S^{n-2}$ and using the inequality (4), we have the lemma as a consequence of the Fubini theorem.

Corollary 4.6. $\left\|f_{i, j}^{*}\right\|_{p} \leqq A_{p}\|f\|_{p}, 1<p \leqq \infty$, where $A_{p}$ is independent of $i, j$ and $f$.

The corollary follows from 3.2(b) just as did the lemma from 3.3, except that the final appeal to the Fubini theorem is replaced by an appeal to the Minkowski inequality for integrals.

COROllary 4.7.

(a) $\int_{I_{n}}\left[\sup _{y>0} P_{y} * f(x)\right] d x \leqq A \int_{I_{n}} f(x) \log ^{+} f(x) d x+A$.

(b) $\left\|\sup _{y>0} P_{y} * f(x)\right\|_{p} \leqq A_{p}\|f\|_{p}, 1<p \leqq \infty$.

The corollary is a consequence of Lemmas 4.4 and 4.5 and Corollary 4.6.

In order to apply the Corollary 4.7 to the Theorem 4.1 , a density argument is needed.

Lemma 4.8. Suppose that $\int_{I_{n}} f(x) \log ^{+} f(x) d x<\infty$.

Then there is a sequence $\left\{f_{m}\right\}$ of continuous functions such that for any $K>0$,

$$
\int_{I_{n}}\left|f-f_{m}\right| \log ^{+}\left|K\left(f-f_{m}\right)\right|<1 / K
$$

if $m$ is sufficiently large.

Proof. Notice that if $u \geqq e$, then

$$
u(\log K u) \leqq(1+\log K)(u \cdot \log u),
$$

so there exists a sufficiently large $N \geqq e$ such that

$$
\int_{f>N} f(x) \log ^{+} K f(x) d x<1 / K .
$$

We may therefore assume that $f$ is bounded on $I_{n}$ by a constant $N$, which may depend on $K$. (Note that we assume $K \geqq 1$.) 
But if $u \leqq N$, then $u \log ^{+} K u \leqq u(\log K N)$, so that if $\left\{f_{m}\right\}$ is a sequence of continuous functions converging to $f$ in the $L^{1}\left(I_{n}\right)$ norm, then

$$
\int_{I_{n}}\left|f-f_{m}\right| \log ^{+}\left|K\left(f-f_{m}\right)\right| \leqq \log (K N) \int_{I_{n}}\left|f-f_{m}\right| \rightarrow 0 .
$$

REMARK. It is possible to make the space of all functions on $I_{n}$ such that

$$
\int_{I_{n}}|f| \log ^{+}|f|<\infty
$$

into a Banach space $L_{\Phi}$ by defining

$$
\|f\|_{\odot}=\inf \left\{\theta: \int_{I_{n}}\left(|f| \theta^{-1}+1\right) \log \left(|f| \theta^{-1}+1\right) \leqq 1\right\} .
$$

Lemma 4.8 could then be placed in the more elegant form: "Continuous functions are dense in $L_{\Phi}$." Also, it can be shown that Lemma 4.5 is equivalent to the norm inequality: $\left\|f_{i, j}^{*}\right\|_{1} \leqq A\|f\|_{\Phi}$. (cf. Zygmund [15, II, Chapter 4, §10].) But in neither case can the alternate form be proved without reference to the original form, and so there seems to be no point in employing the $L_{\Phi}$ norm here.

Proof of Theorem 4.1(a). It is enough to show that $u(x+i y) \rightarrow f(x)$ as $y \rightarrow 0$ restrictedly for a.e. $x$ belonging to an arbitrary bounded set, say

$$
\tilde{I}_{n}=\left\{x \in E_{n}:\left|x_{j}\right| \leqq 1 / 2, j=1, \ldots, n\right\} .
$$

What we must prove is that for any $\varepsilon>0$,

$$
\left|\tilde{I}_{n} \cap\left\{x: \limsup _{y \rightarrow 0}|f(x)-u(x+i y)|>\varepsilon\right\}\right|<\varepsilon .
$$

Write $f=f_{0}+f_{1}$, where $f_{0}$ is supported on $I_{n}$ and $f_{1}$ vanishes on $I_{n}$. Let $g_{0}$ be a continuous function on $I_{n}$ such that if $h_{0}=f_{0}-g_{0}$, then

$$
\int_{I_{n}}\left|h_{0}(x)\right| \log ^{+}\left[\varepsilon^{-2}\left|h_{0}(x)\right|\right] d x<\varepsilon^{2}
$$

It follows from Corollary 4.7 that

$$
\int_{I_{n}}\left[\sup _{y>0}\left|P_{y} * h_{0}(x)\right|\right] d x \leqq A \int_{I_{n}}\left|h_{0}(x)\right| \log ^{+}\left|h_{0}(x)\right| d x+A
$$

and, replacing $h_{0}$ by $\varepsilon^{-2} h_{0}$, it follows from (8) that

$$
\int_{I_{n}}\left[\sup _{y>0}\left|P_{y} * h_{0}(x)\right|\right] d x \leqq A \int_{I_{n}}\left|h_{0}(x)\right| \log ^{+}\left(\varepsilon^{-2}\left|h_{0}(x)\right|\right) d x+\varepsilon^{2} A \leqq 2 A \varepsilon^{2} .
$$

In particular, the set on which $\left|P_{y} * h_{0}(x)\right|>\varepsilon$ has measure less than $\varepsilon^{-1}\left(2 A \varepsilon^{2}\right)$ $=2 A \varepsilon$, and so (7) holds for $u_{h}=P_{y} * h_{0}$.

Since $g_{0}$ is continuous and $P_{y}(t)$ is an approximation to the identity (cf. $\S 1$ ), $P_{y} * g_{0}(x) \rightarrow g_{0}(x)$ a.e. in $\tilde{I}_{n}$ as $y \rightarrow 0$; and certainly (7) holds for $u_{g}=P_{y} * g_{0}$, and so for $P_{y} * f_{0}=u_{0}=u_{h}+u_{g}$. 
A similar argument shows that (7) holds for $u_{1}=P_{y} * f_{1}$. It is necessary to notice only that if $x \in \tilde{I}_{n}, t \notin I_{n}$, then $P_{y}(x-t) \leqq c_{n}$, which is immediate from the estimates of Lemma 4.4. In particular, $\left|P_{y} * f_{1}(x)\right| \leqq c_{n}\left\|f_{1}\right\|_{1}$ if $x \in \tilde{I}_{n}$.

This completes the proof of Theorem 4.1(a).

The proof of 4.1(b) now follows immediately by the identical method.

Proof of Theorem 4.2. On $E_{3}$, define

$$
f^{*}(x)=\sum_{i \leqq j} 2^{-[j+2 i]} f_{i, j}^{*}(x) \text {. }
$$

It follows from Lemma 4.4 that $\sup _{y>0} P_{y} * f(x) \leqq A f^{*}(x)$. Reasoning as in the proof of Theorem 4.1 just completed, we see that to prove Theorem 4.2 , it will be enough to show that for every $s>0$,

$$
m\left(f^{*}\right)(s)=\left|\left\{x: \tilde{f}^{*}(x)>s\right\}\right|<A s^{-1} \int_{E_{3}} f(x) d x .
$$

Let us restrict our attention for the moment to

where

$$
f_{j}^{*}(x)=f_{j, 0}^{*}(x)=\sup _{\varepsilon>0}\left|B_{j}^{\varepsilon}\right|^{-1} \int_{B_{j}^{\varepsilon}} f\left(x_{\top} t\right) d t,
$$

$$
B_{j}^{\varepsilon}=\left\{t=\left(t_{1}, t_{2}, t_{3}\right)=\left(t_{1},|\rho|, \theta\right):\left|t_{1}\right|+|\rho| \leqq 2^{j} \varepsilon,|| t_{1}|-| \rho|| \leqq \varepsilon\right\} .
$$

Partitioning $[0,2 \pi]$ into $N$ equal subintervals, $d_{1}, \ldots, d_{N}$, we can express $B_{j}^{\varepsilon}$ as $\bigcup_{k=1 k}^{n} B_{j}^{\varepsilon}$, where

$$
{ }_{k} B_{j}^{\varepsilon}=B_{j}^{\varepsilon} \bigcap\left\{\left(t_{1},|\rho|, \theta\right): \theta \in d_{k}\right\} .
$$

Setting ${ }_{k} f_{j}^{*}(x)=\sup _{\varepsilon>0}\left|{ }_{k} B_{j}^{\varepsilon}\right|^{-1} \int_{k B_{j}^{\varepsilon}} f(x-t) d t$, it follows that $f_{j}^{*}(x) \leqq\left(1 / N^{*}\right) \sum_{k=1 k}^{N} f_{j}^{*}(x)$. In contrast to the case of a norm inequality, however, we can now conclude only that

$$
m\left(f_{j}^{*}\right)(s) \leqq \sum_{k=1}^{N} m\left({ }_{k} f_{j}^{*}\right)(s) .
$$

We show that for $N=2^{j / 2}$,

$$
m\left({ }_{k} f_{j}^{*}\right)(s) \leqq B s^{-1} \int_{E_{3}} f(x) d x, \quad k=1, \ldots, N
$$

where $B$ is independent of $f, j$ and $k$.

Since (11) holds for maximal functions over rectangular parallelepipeds whose sides have a fixed, but arbitrary, ratio (Lemma 3.2(a)), it will suffice to show that each "wedge" ${ }_{k} B_{j}^{\varepsilon}$ can be covered by a rectangular parallelepiped ${ }_{k} R_{j}^{\varepsilon}$ in such a manner that

$$
\left|{ }_{k} R_{j}^{\varepsilon}\right| /\left.\right|_{k} B_{j}^{\varepsilon} \mid \leqq a,
$$

where $a$ is independent of $j, k$, and $\varepsilon$. 
There is an obvious covering of each ${ }_{k} B_{j}^{\varepsilon}$ by a rectangular parallelepiped ${ }_{k} R_{j}^{\varepsilon}$ whose "length" is a multiple of $2^{j} \varepsilon$ and whose cross-sectional dimensions are multiples of $2^{j} \varepsilon(\sin (\pi / N))$ and $\left[1+c^{\prime} 2^{j}(1-\cos (\pi / N))\right] \varepsilon$.

But then $\left|{ }_{k} R_{j}^{\varepsilon}\right| \leqq a \varepsilon^{3} 2^{2 j} N^{-1}\left[1+c^{\prime} 2^{j}(\pi / N)^{2}\right]$, and, on the other hand, $\left|{ }_{k} B_{j}^{\varepsilon}\right| \geqq$ $a^{\prime} \varepsilon^{3} 2^{2 j} N^{-1}$, so that if $N=2^{j / 2}$, we indeed have (12).

Now (11), together with (10), implies that

$$
m\left(2^{-j / 2} f_{j}^{*}\right)(s) \leqq B s^{-1} \int_{E_{3}} f(x) d x .
$$

Returning now to the general case when $0 \leqq i \leqq j$, and reasoning as above, we find that

$$
m\left(2^{-(j-i) / 2} f_{i, j}^{*}\right)(s) \leqq B s^{-1} \int_{E_{3}} f(x) d x .
$$

(For larger $i,{ }_{k} B_{i, j}$ is less "thin," implying that a smaller value of $N\left(N=2^{(j-i) / 2}\right)$ can be used.) Finally, set

$$
f^{* *}(x)=\sup _{i, j}\left\{2^{-[(3 / 4) j-(1 / 4) i]} f_{i, j}^{*}(x)\right\} .
$$

Then

$$
\begin{aligned}
\left|\left\{x: f^{* *}(x)>s\right\}\right| & \leqq \sum_{i \leqq j}\left|\left\{x: 2^{-(j-i) / 2} f_{i, j}^{*}(x)>2^{(i+j) / 4} s\right\}\right| \\
& \leqq\left(\sum_{i \leqq j} 2^{-(i+j) / 4}\right) B s^{-1} \int_{E_{3}} f(x) d x=B^{\prime} s^{-1} \int_{E_{3}} f(x) d x,
\end{aligned}
$$

where we have used (13). That is, (9) holds for $f^{* *}(x)$. But

$$
\begin{aligned}
\tilde{f}^{*}(x) & =\sum_{i \leqq j} 2^{-[j+2 i]} f_{i, j}^{*}(x) \\
& \leqq \sum_{i, j} 2^{-[(1 / 4) j+(2+(1 / 4))]} f^{* *}(x) \\
& =c f^{* *}(x),
\end{aligned}
$$

and so (9) holds for $f^{*}(x)$, and the theorem is proved.

Remarks. In terms of the Property A defined in $\$ 3$, the point of the proof is that while the conical surfaces $B_{j}^{\varepsilon}$ do not satisfy the property, the wedges ${ }_{k} B_{j}^{\varepsilon}$ do. The difficulty in extending the theorem to the case $n>3$ is that the number $N$ of subsets into which we would have to divide $S^{n-2}$ to achieve (12) increases with $n$. (In fact, $N=\left(2^{j / 2}\right)^{n-2}$.) And any value of $N$ greater than or equal to $2^{j}$ makes the above proof impossible, since then there are no negative powers of $2^{j}$ left over to provide convergence in (14).

5. Compact Jordan algebras. The main result of this section is that any irreducible domain of positivity can be realized as a subset of a simple compact 
Jordan algebra. We set down enough properties of the latter to enable us to prove our convergence theorem in $\S 6$. Any results not attributed explicitly may be found either in the paper of Jordan, von Neumann and Wigner [6] or the book of Braun and Koecher [2].

A Jordan algebra is a commutative, nonassociative, finite-dimensional algebra satisfying the condition:

$$
a\left(b a^{2}\right)=(a b) a^{2}, \quad a, b \in A .
$$

Unless otherwise noted, all Jordan algebras in this section are given over the reals.

For $a \in A$, denote by $L(a)$ the linear transformation on $A$ given by $L(a) b=a b$. A Jordan algebra is said to be compact if the bilinear form $(a, b) \rightarrow \operatorname{Tr}(L(a b))$ is positive definite. As is shown in [8, Chapter $6, \S 4]$, compactness is equivalent to the property of being formally real in the sense that $a^{2}+b^{2}+\cdots=0$ implies $a=b=\cdots$ $=0$. In their original paper [6], Jordan, von Neumann and Wigner referred to formally real Jordan algebras as " $r$-number algebras."

If $A$ is compact, then $A$ has an identity element and $A$ is semisimple. The latter is equivalent, among other things, to the fact that $A$ can be written as the algebraic direct sum $A=A_{1} \oplus \cdots \oplus A_{n}$, where each $A_{j}$ is simple, i.e., has no nontrivial ideals. From our standpoint, the crucial implication of the above algebraic properties is:

THEOREM 5.1. Let $D$ be a domain of positivity.

Then there exists a compact Jordan algebra $A$ such that $D$ is (up to equivalence) the connected component of the identity in

$$
\{y \in A: \operatorname{det} L(y) \neq 0\} \text {. }
$$

Conversely, any such set is a domain of positivity.

For the proof of Theorem 5.1, see [9, Theorems 9 and 11] or [8, Chapter 6, §4]. The inner product making $A$ into a Euclidean space is the one given above: $a \cdot b=\operatorname{Tr}(L(a b))$. The transitive group $G$ on $D$ is identifiable as a subgroup of the group $G L(A)$ of linear automorphisms of $A$.

We note that $D \subset A$ can be defined alternatively as

$$
\{y \in A: L(y) \text { is positive definite }\}
$$

or

$$
\left\{y \in A: y=x^{2}, \operatorname{det} Q(x) \neq 0\right\},
$$

where $Q(x)$ is as defined in $\S 6$.

Let us call a domain of positivity irreducible if it cannot be expressed as a Cartesian product of domains of positivity. It is immediate that the domain $D$ is irreducible if and only if $A$ is simple. For the remainder of this section, $A$ will be assumed to be simple. 
A useful result on the structure of $A$ is the following:

THEOREM $5.2\left(\left[6\right.\right.$, p. 41]). There is a basis for $A$ of the form $\left\{e_{i}, S_{i j}^{\lambda}\right\}, i=1, \ldots, m$; $i<j ; \lambda=1, \ldots, \chi$, where $m$ and $\chi$ depend only on $A$, satisfying:
(a) $e_{i} e_{j}=\delta_{i j} e_{i}$
(b) $e_{i} S_{j k}^{\lambda}=\frac{1}{2}\left(\delta_{i j}+\delta_{i k}\right) S_{j k}^{\lambda}$
(c) $S_{i j}^{\lambda} S_{i j}^{\mu}=\delta_{\lambda \mu}\left(e_{i}+e_{j}\right)$
(d) $S_{i j}^{\lambda} S_{j k}^{\mu}=\sum_{v=1}^{x} A_{i j k}^{\lambda \mu \nu} S_{i k}^{v}$
(e) $S_{i j}^{\lambda} S_{k l}^{\mu}=0, i, j \neq k, l$.

An easy computation reveals that the above is actually an orthogonal basis for $A$ with respect to the inner product $a \cdot b=\operatorname{Tr}(L(a b))$.

It is shown in [6] that a simple compact Jordan algebra is of one of these five types:

(a) $\mathscr{C}_{n}, n=3,4, \ldots$, the algebra generated by the identity $f_{1}$, and $f_{2}, \ldots, f_{n}$ with the multiplication rules $f_{i} f_{j}=\delta_{i j} f_{1}, i, j>1$.

(b) $\mathscr{S}_{m}, m=1,2, \ldots$, the real $n \times n$ symmetric matrices.

(c) $\mathscr{H}_{m}, m=1,2, \ldots$, the complex $n \times n$ hermitian symmetric matrices.

(d) $\mathscr{Q}_{m}, m=1,2, \ldots$, the quaternionic $n \times n$ hermitian symmetric matrices.

(e) $\mathscr{E}$, the exceptional algebra consisting of $3 \times 3$ hermitian matrices over the Cayley numbers.

In cases (b)-(e) the multiplication is given by $a b=\frac{1}{2}(a \circ b+b \circ a)$, where $a \circ b$ denotes ordinary matrix multiplication.

The corresponding domains of positivity, denoted $C_{n}, S_{n}, H_{n}, Q_{n}$ and $E$ are easily seen to be, respectively, the forward light cone and the set of positive definite matrices in each of the matrix algebras.

In the case of the matrix algebras, the basis given in Theorem 5.2 is the obvious one; the value of $\chi$ is, respectively, $1,2,4$ and 8 . Identifying $\mathscr{C}_{n}$ with $E_{n}$ by letting the $f_{i}$ be the usual unit vectors, the two idempotents are

$$
e_{1}=\left((n-1)^{1 / 2}, 1, \ldots, 1\right) / 2(n-1)^{1 / 2}
$$

and $e_{2}=\left((n-1)^{1 / 2},-1, \ldots,-1\right) / 2(n-1)^{1 / 2}$. (And $\chi=n-2$.)

The subgroup $K^{\prime}$ of $G$ which fixes the identity $e$ of $A$ can be shown to coincide with the group of algebra automorphisms of $A$. It is also true that $K^{\prime}$ is a subgroup of the orthogonal group on $A$.

The following is a consequence of a theorem in [2, p. 329].

THEOREM 5.3. Let $K$ be the connected component of the identity in $K^{\prime}$.

Then for every $x \in A$, there exists $k \in K$ such that

$$
k(x)=\sum_{i=1}^{m} a_{i} e_{i}
$$

where the $a_{i}$ are real and the $e_{i}$ are the idempotents of Theorem 5.2. 
The theorem in [2] is stated for another subgroup $K_{0} \subset K^{\prime}$, but an easy argument yields Theorem 5.3. (See the remark at the close of the proof of Lemma 6.6.)

For the matrix domains $\mathscr{S}_{n}, \mathscr{H}_{n}$ and $\mathscr{Q}_{n}$, Theorem 5.3 is the usual result about diagonalization of symmetric matrices; for $\mathscr{C}_{n}$, the group $K$ is $\mathrm{SO}(n-1)$, and 5.3 is nothing more than the statement that $\mathrm{SO}(n-1)$ acts transitively on $S^{n-2}$.

6. The main result. We again begin by stating the main result of the section.

THEOREM 6.1. Let $f$ be a real valued function on the Euclidean space $E_{n}$. Let $P_{y}(x)$ be the Poisson kernel with respect to the irreducible domain of positivity $D \subset E_{n}$. On $T_{D}$, set $u(x+i y)=P_{y} * f(x)$.

Suppose further that one of the following holds:

(a) $f \in L^{1}\left(E_{n}\right)$ and $\int_{B} f(x) \log ^{+} f(x) d x<\infty$ whenever $B$ is bounded;

(b) $f \in L^{p}\left(E_{n}\right)$ for some $p, 1<p \leqq \infty$.

Then $u(x+i y) \rightarrow f(x)$ for a.e. $x \in E_{n}$ as $y \rightarrow 0$ restrictedly.

The theorem will be proved using the correspondence between irreducible domains of positivity and simple Jordan algebras of $\S 5$. Thus we assume that $D$ is the connected component of $e$ in

$$
\{y \in A: \operatorname{det} L(y) \neq 0\},
$$

where $A$ is a simple compact Jordan algebra.

Let us define the so-called quadratic representation on $A$. To any $x \in A$, we associate the linear transformation $Q(x)$ on $A$ given by

$$
Q(x)=2(L(x))^{2}-L\left(x^{2}\right), \text { i.e., } Q(x) w=2 x(x w)-x^{2} w .
$$

We note in passing that the transitive group $G$ on $D$ is the same as

$$
\left\{T \in G L(A): Q(T a)=T Q(a) T^{*}, \text { all } a \in A\right\},
$$

where $T^{*} \in G L(A)$ depends only on $T$ ([8, Chapter 4, §3]).

Consider now the complexification $A+i A$ of the Jordan algebra $A$. We identify $T_{D}$ with $\{x+i y: x \in A, y \in D\}$.

Lemma 6.2 ([10, p. 27], [8, Chapter 6, §5]). For $y \in D$,

$$
K(i y)^{-2}=C^{\prime} \operatorname{det} Q(y) .
$$

In particular, $K(i y)^{-2}$ is a polynomial; the analytic continuation of $K(i y)^{-2}$ vanishes nowhere on $T_{D}$.

It follows from the lemma and from (3), $\S 2$, that for $x+i y \in T_{D}$,

$$
\begin{aligned}
K(x+i y) & =C[\operatorname{det} Q(-i(x+i y))]^{-1 / 2}, \\
P_{y}(x) & =C[\operatorname{det} Q(2 y)]^{1 / 2} /|\operatorname{det} Q(x+i y)| .
\end{aligned}
$$

Now let $y \in D$. By Theorem 5.3 there exists $k \in K$ such that $k(y)=\sum_{i=1}^{m} y_{i} e_{i}$. The condition of $y \in D$ implies in particular that each $y_{i}$ is positive. It is routine to show 
that the condition of restricted convergence, $|y| \mid d(y, \partial D)<a$ is equivalent to the condition

$$
y_{i} / y_{j}<b, i, j=1, \ldots, m
$$

for some constant $b$. Denote by $\bar{y}$ the smallest of the $y_{j}$.

Lemma 6.3. Let $x \in A$, and suppose that $y \in D$ satisfies (2).

Then $P_{y}(x) \leqq B P_{i j e}(x)$, where $e \in A$ is the identity and $B$ depends only on $b$.

Proof. A direct computation reveals that for any $z$, the matrix of $Q(z)$ with respect to the basis $\left\{e_{i}, S_{i j}^{\lambda}\right\}$ is symmetric. We denote this matrix by $Q(z)$ also. Since any $k \in K$ is orthogonal, and, as is easily checked, $Q(k(z))=k Q(z) k^{-1}$, we may assume that $y=\sum_{i=1}^{m} y_{i} e_{i}$. (The $y_{i}$ will be positive.)

The basis elements $e_{i}$ and $S_{i j}^{\lambda}$ are easily seen to be eigenvectors of $Q\left(\sum_{i=1}^{m} y_{i} e_{i}\right)$ with the respective eigenvalues $y_{i}^{2}$ and $y_{i} y_{j}$, so that as a matrix, $Q(y)$ is diagonal and

$$
\operatorname{det} Q(y)=\prod_{i=1}^{m} y_{i}^{2} \prod_{i<j}\left(y_{i} y_{j}\right)^{x}=\left(\prod_{i=1}^{m} y_{i}\right)^{x(m-1)+2} .
$$

It follows in particular that

$$
\operatorname{det} Q(y) \leqq b^{2 n} \operatorname{det} Q(\bar{y} e),
$$

(recalling from 5.2 that $n=\operatorname{dim} A=\frac{1}{2} m(m-1) \chi+m$ ).

It thus follows from (1) that the proof will be complete if we can establish:

Lemma 6.4. Let $y=\sum_{j=1}^{m} y_{j} e_{j} \in D$ and $x \in A$. Then $\left(\partial / \partial y_{1}\right)|\operatorname{det} Q(x+i y)| \geqq 0$.

Proof. Set $y_{0}=e_{1}+\sum_{j=2}^{m} y_{j} e_{j}$. By the fundamental identity for the quadratic representation $[8$, p. 64],

$$
\begin{aligned}
Q(x+i y) & =Q\left(y_{0}^{-1 / 2}\right)^{-1}\left[Q\left(Q\left(y_{0}^{-1 / 2}\right) x+i Q\left(y_{0}^{-1 / 2}\right) y\right)\right] Q\left(y_{0}^{-1 / 2}\right)^{-1} \\
& =Q\left(y_{0}^{-1 / 2}\right)^{-1}\left[Q\left(x_{0}+i\left(y_{1} e_{1}+\sum_{j=2}^{m} e_{j}\right)\right)\right] Q\left(y_{0}^{-1 / 2}\right)^{-1},
\end{aligned}
$$

where $x_{0}=Q\left(y_{0}^{-1 / 2}\right) x$ is arbitrary since $x$ is. Moreover, $x_{0}=c_{1} e_{1}+\sum_{j, \lambda} d_{1 j}^{\lambda} S_{1 j}^{\lambda}+x_{1}$, where $x_{1} \in A_{1}=\left\{x \in A: e_{1} x=0\right\}$. And $A_{1}$ is a subalgebra of $A$, so [2, p. 329] there exists $w_{1} \in A_{1}$ such that $w_{1}^{2}=\sum_{j=2}^{m} e_{j}$ and $Q\left(w_{1}\right) x_{1}=\sum_{j=2}^{m} c_{j} e_{j}$. Setting $w=w_{1}+e_{1}$, we have $w^{2}=e$ (which implies that $Q(w)$ is an automorphism of $A$ ) and

$$
\begin{aligned}
Q(w)\left(x_{0}+i\left(y_{1} e_{1}+\sum_{j=2}^{m} e_{j}\right)\right) & =\sum_{j=1}^{m} c_{j} e_{j}+\sum_{j, \lambda} c_{1 j}^{\lambda} S_{1 j}^{\lambda}+i\left(y_{1} e_{1}+\sum_{j=2}^{m} e_{j}\right) \\
& =\sum_{j=1}^{m} z_{j} e_{j}+\sum_{j, \lambda} c_{1 j}^{\lambda} S_{1 j}^{\lambda} .
\end{aligned}
$$

On the other hand,

$$
\operatorname{det} Q\left(\sum_{j} z_{j} e_{j}+\sum_{j, \lambda} c_{1 j}^{\lambda} S_{1 j}^{\lambda}\right)=\left[z_{1} \cdots z_{m}-\sum_{j, \lambda}\left(c_{1 j}^{\lambda}\right)^{2} \prod_{k=2 ; k \neq j}^{m} z_{k}\right]^{2 n / m} .
$$


This is directly verifiable for each class of nonexceptional compact Jordan algebras. It can be shown to hold in the exceptional case by a tedious and inelegant method involving the multiplication table for the Cayley numbers and the fact that $\operatorname{det} Q(x)=[R(x)]^{2 n / m}$, where $R$ is a homogeneous polynomial of degree $m$ in the coefficients of $x$.

Finally, it fo!lows from (4) and (5) that

$$
\begin{aligned}
\frac{\partial}{\partial y_{1}}(\mid \operatorname{det} & \left.\left.Q\left(x_{0}+i\left(y_{1} e_{1}+\sum_{j=2}^{m} e_{j}\right)\right)\right|^{m / n}\right) \\
& =2 \operatorname{Re}\left[\left(i z_{2} \cdots z_{m}\right)\left(\bar{z}_{1} \cdots \bar{z}_{m}-\sum_{j, \lambda}\left(c_{1 j}^{\lambda}\right)^{2} \prod_{k=2 ; k \neq j}^{m} \bar{z}_{k}\right)\right] \\
& =2\left[y_{1}\left|z_{2}\right|^{2} \cdots\left|z_{m}\right|^{2}+\sum_{j, \lambda}\left(c_{1 j}^{\lambda}\right)^{2} \prod_{k=2 ; k \neq j}^{m}\left|z_{k}\right|^{2}\right] \geqq 0 .
\end{aligned}
$$

This, together with (3a), completes the proof of the lemma, and so also that of Lemma 6.3.

Corollary 6.5. $|\operatorname{det} Q(x+i \bar{y} e)|^{2} \geqq C|\operatorname{det} Q(x+i y)|^{2}$.

Proof. Let $y^{*}=\max \left\{y_{1}, \ldots, y_{n}\right\}$. It follows from (1) that $y^{*}<b \bar{y}$, and so, using the method of the lemma, we have (noting that $b \geqq 1$ )

$$
|\operatorname{det} Q(x+i y)|^{2} \leqq\left|\operatorname{det} Q\left(x+i y^{*} e\right)\right|^{2} \leqq b^{2 n}|\operatorname{det} Q(x+i \bar{y} e)|^{2} .
$$

It is a consequence of 6.3 and 6.5 that $P_{y}(x)$ and $P_{\bar{y} e}(x)$ are comparable, that is, that restricted convergence is equivalent to convergence along the ray passing through the identity.

From here on, we assume $y=\bar{y} e=y e$, again abusing notation by using $y$ to denote both a member of $D \subset A$ and a positive number.

Before proceeding to the proof of 6.1 , two preliminary results are needed concerning integration over $A$. As we saw in Theorem 5.3, any $x \in A$ can be diagonalized by some $k \in K$, the connected component of the identity in the automorphism group of $A$; that is, $k(x)=\sum_{i=1}^{m} t_{i} e_{i}$, where the $t_{i}$ are real. We write this sum as $d(t), t \in E_{m}$. Now letting $M$ be the subgroup of $K$ which takes each $x \in A$ of the form $x=d(t)$ into itself, and $E_{m}^{\prime}=\left\{\left(t_{1}, \ldots, t_{m}\right): t_{1} \geqq t_{2} \geqq \cdots \geqq t_{m}\right\}$, there is a bijection $E_{m}^{\prime} \times K \mid M \rightarrow A$, which is given by $(t,[k]) \rightarrow k(d(t))$. There is an obvious Euclidean measure $d x$ on $A$ corresponding to the orthogonal basis $\left\{e_{i}, S_{i j}^{\lambda}\right\}$.

LEMma 6.6. Writing $x=k(d(t)), d x=\Delta(t) d t d k$, where $d t$ is Euclidean measure on $E_{m}^{\prime}, d k$ is the induced measure on the coset manifold $K / M$, and

$$
\Delta\left(t_{1}, \ldots, t_{m}\right)=C_{m} \prod_{j>i}^{m}\left|t_{i}-t_{j}\right|^{x} .
$$

The formula does not, of course, hold on the set of measure zero consisting of $x \in A$ with some equal eigenvalues, (where we refer to the $t_{i}$ as eigenvalues). 
Proof. We wish to find the Jacobian of the map $\eta: E_{m}^{\prime} \times K \mid M \rightarrow A$ at the point $(t,[k])$. Since each $k^{\prime} \in K$ is orthogonal, the Jacobian of the map $x \rightarrow k^{\prime}(x)$ of $A$ to $A$ is 1 , and so we can assume $[k] \in K / M$ to be the identity.

Denote by $\mathfrak{f}$ and $\mathfrak{m}$ the Lie algebras of $K$ and $M$ respectively. Then $\mathfrak{t}-\mathfrak{m}$ can be regarded as the tangent space to $K / M$ at the identity. It can be shown [8, Chapter 4 , $\S 5]$, that $\mathfrak{f}=\{[L(a), L(b)]: a, b \in A\}$, where $\left[T_{1}, T_{2}\right]=T_{1} T_{2}-T_{2} T_{1}$ as usual. It follows from the multiplication rules of Theorem 5.2 that the following is a basis for $\mathfrak{l}-\mathfrak{m}$ :

$$
\left\{\left[L\left(e_{i}-e_{j}\right), L\left(S_{i j}^{\lambda}\right)\right]: i>j=1, \ldots, m ; \lambda=1, \ldots, \chi\right\} .
$$

Now identify the tangent space to $E_{m}^{\prime}$ at $t$ with $E_{m}$ and the tangent space at $d(t) \in A$ with $A$. It follows that $\delta \eta: E_{m} \times \mathfrak{t}-\mathfrak{m} \rightarrow A$ is given by

$$
\delta \eta(\delta t,[L(a), L(b)])=d(\delta t)+[L(a), L(b)](d(t)) .
$$

To evaluate the Jacobian, it is enough to notice that

$$
\left[L\left(e_{i}-e_{j}\right), L\left(S_{i j}^{\lambda}\right)\right]\left(\sum_{k} t_{k} e_{k}\right)=\left(e_{i}-e_{j}\right)\left(\frac{t_{i}+t_{j}}{2}\right) S_{i j}^{\lambda}+S_{i j}^{\lambda}\left(\frac{t_{i}-t_{j}}{2}\right)=0+S_{i j}^{\lambda}\left(\frac{t_{i}-t_{j}}{2}\right) .
$$

This completes the proof of Lemma 6.6.

We remark that the isomorphism, $\delta \eta$, of tangent spaces enables us to establish Theorem 5.3 from a similar theorem in [2].

We now estimate the volume in $A$ of the set of elements whose eigenvalues lie in the rectangular set

$$
R_{(j)}^{\varepsilon}=\left\{t \in E_{m}:\left|t_{i}\right| \leqq 2^{j_{i}} \varepsilon\right\} .
$$

LEMMA 6.7. Suppose that $j_{1} \geqq j_{2} \geqq \cdots \geqq j_{m} \geqq 0$. Then

$$
\int_{R_{(j)}^{\varepsilon}} \prod_{j>i}^{m}\left|t_{i}-t_{j}\right|^{x} d t \sim \varepsilon^{n} 2^{j_{1}[(m-1) x+1]+j_{2}[(m-2) x+1]+\cdots+j_{m}},
$$

where $n$ is the dimension of $A$.

Proof. Homogeneity of degree $n$ follows from the identity $n=\frac{1}{2} m(m-1) \chi+m$. We now assume that $\varepsilon=1$. Writing

$$
\Delta(t)=\prod\left|t_{i}-t_{j}\right|^{x}, R H S \geqq B\left|R_{(j)}^{1}\right| \sup \left\{\Delta(t): t \in R_{(j)}^{1}\right\} \geqq B(L H S) .
$$

We prove that $L H S \geqq B^{\prime}(R H S)(*)$ by induction on $m$. Suppose for now that $\chi=1$. When $m=2$,

$$
\begin{aligned}
\int_{R_{(j)}^{1}}\left|t_{i}-t_{j}\right| d t & =I_{2}=\left[\left(2^{j_{1}}+2^{j_{2}}\right)^{3}-\left(2^{j_{1}}-2^{j_{2}}\right)^{3}\right] / 3 \\
& \geqq 2\left(2^{2 j_{1}+j_{2}}\right) .
\end{aligned}
$$


Now suppose that $(*)$ holds for $m-1$. Then

$$
\begin{aligned}
& I_{m} \geqq \int_{(3 / 4) 2^{j_{1}}}^{2^{f_{1}}} \int_{-2^{j_{2}-1}}^{2^{f_{2}-1}} \cdots \int_{-2^{j_{m}-1}}^{2^{f_{m}-1}} \Delta(t) d t \\
& \geqq \int_{(3 / 4) 2^{j_{1}}}^{2^{j_{1}}}\left[\inf _{t_{2} \cdots t_{n}} \prod_{k=2}^{m}\left|t_{1}-t_{k}\right|\right] d t_{1}\left(2^{-m(m-1) / 2} I_{m-1}\right) \\
& \geqq\left(2^{j_{1}} / 4\right)\left(2^{j_{1}} / 4\right)^{m-1}\left(2^{-m(m-1) / 2} I_{m-1}\right) \geqq B^{\prime} 2^{m j_{1}+(m-1) j_{2}+\cdots+j_{m}}
\end{aligned}
$$

where we have used the homogeneity property,

$$
I_{m-1}\left(j_{2}, \ldots, j_{n}\right)=2^{m(m-1) / 2} I_{m-1}\left(j_{2}-1, \ldots, j_{m}-1\right) .
$$

The extension to the case $\chi>1$ is obvious.

We are now able to prove Theorem 6.1 just as we did Theorem 4.1. By Lemma 6.3 , we may assume $y \in D$ to be of the form $y e, y>0$. Once more, $y$ is used to denote both a member of $D$ and a positive number. For any $x \in A$ and $k \in K$, $k(x+i y)=k(x)+i y$, since $k(e)=e$. Therefore, choosing $k$ so that $k(x)=\sum_{i=1}^{m} t_{i} e_{i}$, we have

$$
K(x+i y)=C[\operatorname{det} Q(-i(x+i y))]^{-1 / 2}=C\left[\prod_{i=1}^{m}\left(y-i t_{i}\right)\right]^{-(x(m-1)+2) / 2},
$$

$$
P_{y}(x)=C^{\prime} y^{n} /\left[\prod_{i=1}^{m}\left(y^{2}+t_{i}^{2}\right)\right]^{(x(m-1)+2) / 2},
$$

where we use again the fact that $n=\operatorname{dim} A=\frac{1}{2} m(m-1) \chi+m$, and notice that $P_{y}(x)$ has the correct homogeneity of degree $-n$.

As before, we write $R_{(j)}^{\varepsilon}=\left\{t \in E_{m}:\left|t_{i}\right| \leqq 2^{j_{i}} \varepsilon\right\}$. Assume that $j_{1} \geqq \cdots \geqq j_{m}$.

DEFINITION.

$$
B_{(j)}^{\varepsilon}=\left\{x \in A: x=k(d(t)), t \in R_{(j)}^{\varepsilon},[k] \in K / M\right\} .
$$

For a function $f$ on $A$,

$$
\begin{aligned}
& f_{(j)}^{\varepsilon}(x)=\left|B_{(j)}^{\varepsilon}\right|^{-1} \int_{B_{(j)}^{\varepsilon}} f(x-u) d u, \\
& f_{(j)}^{*}(x)=\sup _{\varepsilon>0} f_{(j)}^{\varepsilon}(x) .
\end{aligned}
$$

Lemma 6.8. Let $y=y e$ and $x \in A$. Then

$$
P_{y} * f(x) \leqq C \sum_{j_{1} \geqq \cdots \geqq j_{m}} 2^{-\left[j_{1}+(x+1) j_{2}+\cdots+((m-1) x+1) j_{m}\right]} f_{(j)}^{*}(x)
$$

Proof. The proof follows exactly as did the proof of Lemma 4.4 in the case of $C_{n}$. A few observations are needed to see that this is so. First of all,

$$
P_{y}(x)=P_{k(y)}(k(x))=(\text { for } y=y e) P_{y}(k(x)) \text {. }
$$


Therefore, if

$$
\begin{aligned}
x & \in B_{j_{1}, \ldots, j_{m}}^{y}-B_{j_{1}-1, \ldots, j_{m}-1}^{y}=F_{j_{1}, \ldots, j_{m}}^{y}, \\
P_{y}(x) & \leqq c^{\prime} y^{n} / 2^{\left[j_{1}+\cdots+j_{m}\right][x(m-1)+2]} y^{2 n}
\end{aligned}
$$

while on the other hand, from Lemma 6.7,

so that

$$
\left|B_{j_{1}, \ldots, j_{m}}^{y}\right| \leqq c y^{n} 2^{j_{1}[(m-1) x+1]+j_{2}[(m-2) x+1]+\cdots+j_{m}},
$$

$$
P_{y}(x) \cdot\left|B_{(j)}^{y}\right| \leqq 2^{-\left[j_{1}+(x+1) j_{2}+\cdots+((m-1) x+1) j_{m}\right]} .
$$

Integration over all $F_{(j)}^{y}$ completes the proof of Lemma 6.8.

We now proceed by the method of rotations. The place of

$$
S^{n-2}(=S O(n-1) / S O(n-2))
$$

is now taken by $K / M$.

LemMA 6.9. Suppose that $f$ is supported on $I=I_{n}$, the unit cube in $A$, and that $f_{(j)}^{*}$ be the maximal function of Lemma 6.8. Then

$$
\int_{I} f_{(j)}^{*}(x) d x \leqq B \int_{I} f(x) \log ^{+} f(x) d x+B,
$$

where $B$ is independent of $(j)$ and $f$.

Proof.

$$
f_{(j)}^{*}(x)=\sup _{\varepsilon>0}\left|B_{(j)}^{\varepsilon}\right|^{-1} \int_{K / M} d[k] \int_{R_{(j)}^{\varepsilon}} f(x-k(d(t))) \Delta(t) d t .
$$

But it follows from Lemma 6.7 that

$$
\left|B_{(j)}^{\varepsilon}\right|^{-1} \leqq \varepsilon^{-n} 2^{-\left[j_{1}[(m-1) x+1]+j_{2}[(m-2) x+1]+\cdots+j_{m}\right]} .
$$

Also,

$$
\sup \left\{\Delta(t): t \in R_{(j)}^{\varepsilon}\right\} \leqq \varepsilon^{n-m} 2^{j_{1}(m-1) x+j_{2}(m-2) x+\cdots+j_{m-1} x}
$$

Therefore,

$$
f_{(j)}^{*}(x) \leqq c \sup _{\varepsilon>0} \int_{K / M} d[k]\left[\varepsilon^{-m} 2^{-\left[j_{1}+\cdots+j_{m}\right]} \int_{R_{(j)}^{\varepsilon}} f(x-k(d(t))) d t\right] .
$$

Denote by $\bar{f}_{(j)}(x ;[k])$ the supremum over $\varepsilon>0$ of the inner term in the last expression. For each fixed $[k] \in K / M, \bar{f}_{(j)}(x ;[k])$ is a rectangular maximal function, and so by Lemma 3.3,

$$
\int_{x-k(d(t)) \in I} \bar{f}_{(j)}(x-k(d(t)) ;[k]) d t \leqq C^{\prime} \int_{\ldots \in I} f(x-k(d(t))) \log ^{+} f(x-k(d(t))) d t+C^{\prime} .
$$

Integrating now over all $n$-dimensional varieties parallel to

$$
V_{x,[k]}=\left\{x+k(d(t)): t \in E_{m}\right\},
$$

i.e., along the orthogonal complement of the subspace $V_{0,[k]}$, we have

$$
\int_{I} \bar{f}_{(j)}(x ;[k]) d x \leqq A \int_{I} f(x) \log ^{+} f(x) d x+A .
$$


Integrating finally over all $[k] \in K / M$, we complete the proof of 6.9. Just as in $\S 4$, we have also

Corollary 6.10. $\left\|f_{(j)}^{*}\right\|_{p} \leqq A_{p}\|f\|_{p}, 1<p<\infty$, where $A_{p}$ is independent of $f$ and of $(j)$.

The proof of Theorem 6.1 is now a consequence of the method of proof of Theorem 4.1, together with Lemmas 6.8 and 6.9 and Corollary 6.10.

7. Almost everywhere convergence on product domains; nontangential convergence. In this section, we derive the expected results about a.e. convergence on tube domains over products of irreducible domains of positivity. Also, the case of restricted nontangential convergence to the boundary is considered.

Suppose now that $D=D_{1} \times \cdots \times D_{k}$ is a Cartesian product of irreducible domains of positivity. By Theorem 5.1 and the fact that a compact Jordan algebra is semisimple, and thus the direct sum of simple Jordan algebras, it can be shown that any domain of positivity is of the above form. In what follows, there will be no loss of generality in assuming that $k=2$.

We notice first that

$$
K\left(z_{1}, z_{2}\right)=\int_{D_{1} \times D_{2}} e^{2 \pi i\left(z_{1}, z_{2}\right) \cdot\left(t_{1}, t_{2}\right)} d t=K\left(z_{1}\right) K\left(z_{2}\right),
$$

so that $P_{\left(y_{1}, y_{2}\right)}\left(x_{1}, x_{2}\right)=P_{y_{1}}\left(x_{1}\right) P_{y_{2}}\left(x_{2}\right)$.

Next, because $\partial\left(D_{1} \times D_{2}\right)=D_{1} \times \partial D_{2} \cup \partial D_{1} \times D_{2}$, it follows that restricted convergence of $\left(y_{1}, y_{2}\right)$ to $(0,0)$ requires the restricted convergence of $y_{1}$ and $y_{2}$ to 0 separately. We know from Lemma 6.3 that for our purposes, $y_{1}$ and $y_{2}$ can each be assumed to converge to 0 along a single ray. We again denote by $y_{1}$ and $y_{2}$ both the vectors and the parameters on the respective rays. The condition of restricted convergence now implies further that

$$
c \leqq y_{1} / y_{2} \leqq c^{\prime},
$$

for some constants $c$ and $c^{\prime}$. It is clear now from the estimates of the Poisson kernel in Lemma 6.8 that, given the condition of restricted convergence, $\left(y_{1}, y_{2}\right)$ can be assumed to be of the form $(y, y)$, where $y$ is the parameter along the respective rays.

Following now the procedure of 6.8 , we conclude that

$$
P_{(y, y)} * f\left(x_{1}, x_{2}\right) \leqq A \sum_{(j)} 2^{-\left(j_{1}+\cdots\right)} f_{(j)}^{*}\left(x_{1}, x_{2}\right),
$$

where $(j)=\left(j_{1}, \ldots, j_{m_{1}} ; j_{m_{1}+1}, \ldots, j_{m_{1}+m_{2}}\right)$ and $f_{(j)}^{*}$ is a maximal function over the Cartesian product of two surfaces of the type $B_{(j)}^{\varepsilon}$.

Application of the method of rotations leads to the expression of $f_{(j)}^{*}$ in terms of an $\left(m_{1}+m_{2}\right)$-dimensional rectangular maximal function.

The method of Proof of Theorem 4.1 finally leads us to

THEOREM 7.1. The results of Theorem 6.1 hold for arbitrary domains of positivity. 
Notice that the only irreducible domains of positivity in three dimensions or less are (up to equivalence) the positive halfline in $E_{1}$ and the light cone $C_{3}$ in $E_{3}$. The method of proof of Theorem 4.2 leads to a result which can be stated in the following form:

THEOREM 7.2. Let $D$ be a Cartesian product of irreducible domains of positivity whose dimensions are at most 3 . Let $f$ be a real-valued function on the appropriate Euclidean space $E_{N}$ and suppose that $f \in L^{p}\left(E_{N}\right)$ for some $p \geqq 1$.

Then $P_{y} * f(x) \rightarrow f(x)$ a.e. on $E_{N}$ as $y \rightarrow 0$ restrictedly.

Until now, we have considered convergence of $x+i y \in T_{D}$ to $x \in E_{n}$ as $y \rightarrow 0$ restrictedly. A more general type of convergence, which is often more natural to consider, (cf. [15, II, Chapter 14]) is restricted, nontangential convergence. We say that $x+i y \in T_{D}$ converges to $X \in E_{n}$ restrictedly and nontangentially if $y \rightarrow 0$ restrictedly and there exists a constant $c$ so that $|X-x|<c|y|$.

THEOREM 7.3. Under the assumptions of Theorem 6.1 , and even if $D$ is an arbitrary domain of positivity,

$$
u(x+i y) \rightarrow f(X) \quad \text { as } \quad x+i y \rightarrow X
$$

restrictedly and nontangentially for a.e. $X \in E_{n}$.

In light of the proof of Theorems 6.1 and 7.1, it will be enough to consider the case when $D$ is irreducible, and to prove in this case:

Lemma 7.4. $P_{y}(x) \leqq C P_{y}(X)$, where $C$ is independent of $x, X$ and $y$.

Proof. As in the proof of Theorem 6.1, we can consider $D$ as a subset of the simple compact Jordan algebra $A$ and assume that $y$ is of the form $y e, y>0, e$ the identity of $A$.

Supposing that $k(x)=\sum_{i=1}^{m} x_{i} e_{i}, k^{\prime}(X)=\sum_{i=1}^{m} X_{i} e_{1}$ for appropriate $k, k^{\prime} \in K$ it follows from (6), $\$ 6$ that Lemma 7.4 is equivalent to

$$
\prod_{i=1}^{m}\left(y^{2}+X_{i}^{2}\right) \leqq C^{\prime} \prod_{i=1}^{m}\left(y^{2}+x_{i}^{2}\right) .
$$

In particular, it suffices to show that for some ordering of the $x_{i}$ and $X_{i}$,

$$
y^{2}+X_{i}^{2} \leqq C^{\prime \prime}\left(y^{2}+x_{i}^{2}\right), \quad i=1, \ldots, m .
$$

Recalling the definition of the inner product in $A$, we see that the condition of nontangential convergence becomes

$$
\operatorname{Tr}\left(L[x-X]^{2}\right) \leqq c^{2} y^{2} .
$$

Moreover, since $L(k(X))=k L(X) k^{-1}$, we may assume that $X=\sum X_{i} e_{i}$. Consider now the expression $\operatorname{Tr}\left(L[k(x)-X]^{2}\right)$ as a function on $K$. Considering the action of the Lie algebra of $K$ on $A$, it is not hard to show that this function has its critical points at values of $k$ for which $k(x)=\sum x_{i} e_{i}$. (I thank Professor J. Simons for this 
argument.) In particular, the function attains its minimum at such a value, and so there is an ordering of the $x_{i}$ and $X_{i}$ such that

$$
\operatorname{Tr}\left(L\left[\sum\left(x_{i}-X_{i}\right) e_{i}\right]^{2}\right) \leqq c^{2} y^{2} .
$$

Computing the trace with respect to the standard basis of Theorem 5.2, it is easy to see that for any $t_{1}, \ldots, t_{m}$,

$$
\operatorname{Tr}\left(L\left[\sum_{i=1}^{m} t_{i} e_{i}\right]^{2}\right)=\left[1+\frac{1}{2} \chi(m-1)\right] \sum_{i=1}^{m} t_{i}^{2} .
$$

It therefore follows from (2) that $\sum\left(x_{i}-X_{i}\right)^{2} \leqq c^{2} y^{2}$, and, in particular, that $\left(x_{i}-X_{i}\right)^{2} \leqq c^{2} y^{2}, i=1, \ldots, m$. Finally, considering separately the cases when $\left|X_{i}\right|>2 c y$ and $\left|X_{i}\right| \leqq 2 c y$, we establish (1). This completes the proof of the lemma, and thus of the theorem.

Remarks. It would be interesting to find an intrinsic definition of restricted nontangential convergence on a tube domain, e.g., in terms of invariance under quasi-analytic automorphisms of the domain. This might prove useful in solving the problem of characterizing convergence of Poisson integrals on the bounded symmetric domains of tube type.

8. Concluding remarks. We conclude by discussing in detail the essentially realvariable problem of the obstacle standing in the way of a proof of a.e. convergence for Poisson integrals of $L^{1}$ functions. The method of rotations in its continuous form is unsuitable because, unlike $L^{p}$ and $L\left(\log ^{+} L\right)$ norms, weak norms are not subadditive. That is, if $m(g)(s)=|\{x: g(x)>s\}|$, we do not have the inequality, $m\left(g_{1}+g_{2}\right)(s) \leqq m\left(g_{1}\right)(s)+m\left(g_{2}\right)(s)$; the most that can be said is that $m\left(g_{1}+g_{2}\right)(s)$ $\leqq 2\left[m\left(g_{1}\right)(s)+m\left(g_{2}\right)(s)\right]$. Using the latter inequality, we made what seems to be the best possible use of a discrete method of rotations in proving Theorem 4.2.

What is needed for the $L^{1}$ case when $n>3$ is a proof that

$$
m\left(f_{(j)}^{*}\right)(s)<(A / s)\|f\|_{1},
$$

where $A$ is independent of $(j)$ as well as of $f .\left(f_{(j)}^{*}\right.$ is the maximal function over "conical" surfaces defined in $\$ 4$ and 6.) As has been mentioned, this is the idea of the proof of the $L^{1}$ result for $T_{\text {octant }}$ in [12]. The problem is that the sets $B_{(j)}^{\varepsilon}$ do not satisfy the Property A set forth in $\S 3$.

Consider, for example, the sets

$$
B_{j}=\left\{\left(x_{1}, r\right), r \in E_{n-1}:\left|x_{1}\right|+|r| \leqq 1,|| x_{1}|-| r|| \leqq 2^{-j}\right\}
$$

as $j$ increases without limit. Letting $\bar{B}_{j}$ be the union of all sets similarly oriented and congruent to $B_{j}$ meeting $B_{j}$, we see that $\left|B_{j}\right| \rightarrow 0$ while $\left|\bar{B}_{j}\right| \geqq a>0$. That is, sets of the form $B_{j}$ do not satisfy Property A.

Nevertheless, it appears that the answer to the question of a.e. convergence for Poisson integrals of $L^{1}$ functions is affirmative. For instance, the hypothesis that 
$f \in L\left(\log ^{+} L\right)$ locally gives us more than we need, implying as it does that

$$
\sup _{y>0} P_{y} * f(x)
$$

is locally integrable. Moreover, there is no indication as to why the exceptional case in 3 dimensions, when a.e. convergence does hold for Poisson integrals of $L^{1}$ functions, should be exceptional.

A proof of the affirmative, involving as it probably does verification of (1), raises the interesting question of the identity of the class of sets which can be indexed according to shape by, say, $n$-tuples $(j)$ of integers, and whose corresponding maximal functions satisfy (1).

\title{
REFERENCES
}

1. S. Bochner, Group invariance of Cauchy's formula in several variables, Ann. of Math. (2) 45 (1944), 686-707.

2. H. Braun and M. Koecher, Jordan-Algebren, Grundlehren der mathematischen Wissenschaften, Band 128, Springer-Verlag, Berlin, 1966.

3. A. P. Calderón and A. Zygmund, On singular integrals, Amer. J. Math. 78 (1956), 289-309.

4. G. H. Hardy and J. E. Littlewood, A maximal theorem with function-theoretic applications, Acta Math. 54 (1930), 81-116.

5. B. Jessen, J. Marcinkiewicz and A. Zygmund, Note on the differentiability of multiple integrals, Fund. Math. 25 (1935), 217-234.

6. P. Jordan, J. von Neumann and E. Wigner, Quantum mechanical formalism, Ann. of Math. (2) 35 (1934), $29-64$.

7. M. Koecher, Analysis in reellen Jordan-Algebren, Nachr. Akad. Wiss. Gottingen IIa (1958), 67-74.

8. - Jordan algebras and their applications (mimeographed), University of Minnesota, Minneapolis, 1962.

9. A. Koranyi, A Poisson integral for homogeneous wedge domains, J. Analyse Math. 14 (1965), 275-84.

10. - Domains of positivity, mimeographed notes.

11. - On the boundary values of holomorphic functions in wedge domains, Bull. Amer. Math. Soc. 69 (1963), 475-480.

12. J. Marcinkiewicz and A. Zygmund, On the summability of double Fourier series, Fund. Math. 32 (1939), 112-132.

13. O. S. Rothaus, Domains of positivity, Abh. Math. Sem. Hamburg 24 (1960), 189-235.

14. E. M. Stein, G. Weiss and M. Weiss, $H^{p}$ classes of holomorphic functions in tube domains, Proc. Nat. Acad. Sci. U.S.A. 52 (1964), 1035-1039.

15. A. Zygmund, Trigonometric series, 2nd ed., Cambridge Univ. Press, New York, 1959.

16. N. Wiener, The ergodic theorem, Duke Math. J. 5 (1939), 1-18.

\author{
Princeton University, \\ Princeton, New Jersey \\ INSTITUTE FOR DefENSE ANAlyses, \\ Princeton, New Jersey
}

